\title{
Early Experience with Autologous Intrastriatal Adrenal Medulla/Nerve Cografting in Parkinson's Disease
}

\author{
R.L. Watts, A. Freeman, S. Graham and R.A.E. Bakay \\ Departments of Neurology, Surgery and Neurosurgery, \\ Emory University, Atlanta, GA 30322, USA
}

\section{INTRODUCTION}

Intrastriatal grafting of autologous adrenal medullary tissue has yielded modest benefits in Parkinson's disease (PD) patients /1/. Parallel studies in non-human primates with experimental parkinsonism have shown that cografts of autologous adrenal medulla and peripheral nerve yield greater behavioral improvement and graft survival $/ 1 /$. Hence, over the past year we have performed intrastriatal implantation of autologous adrenal medulla/intercostal nerve cografts in 3 patients with advanced PD and report our early experience here.

\section{METHODS}

Three patients were selected and evaluated using the Core Assessment Program for Intracerebral Transplantation (CAPIT) protocol $/ 2 /$. They were required to have advanced idiopathic PD (Hoehn \& Yahr Stage 4 or 4) that was no longer responding adequately to antiparkinsonian medications, be in good general health, have 2 functioning adrenal glands, and give informed consent. Patients were maintained on an optimal medication regimen and 3 or more baseline evaluations were performed over the 36 months preceding surgery. The core of the CAPIT evaluation consisted of "off" (12 hours after last medications) and "on" application of the Unified Parkinson Disease Rating Scale (UPDRS), timed motor performance tests (hand pronation-supination test, hand/arm movement between 2 points, finger dexterity, and standwalk-sit test), an L-dopa response test, and brain MRI scanning pre-operatively and at 3 month intervals post-operatively. We also performed electrophysiologic movement time/reaction studies requiring whole arm movements using a computerized touchpad device $/ 3 /$.

We chose 3 targets in the right striatum ( 2 in the caudate and 1 in the putamen) for MR and/or CT-guided stereotaxic implantation of the cografts. A right adrenalectomy was performed via a flank/retroperitoneal approach, and a $3-5 \mathrm{~cm}$ piece of intercostal nerve was harvested from a portion of the 11th rib removed during adrenalectomy. Microdissection removed adrenal cortex and epineurium, and 0.6-0.8 mm diameter $\times 3$ 3-5 mm length portions of medullary tissue were mixed with portions of minced nerve and loaded into a stereotaxic implantation cannula.

\section{RESULTS}

The three PD patients were males, aged 55, 45 , and 53 , and their duration of disease was 12 , 8 and 16 years, respectively. Pre-operative Hoehn \& Yahr stages "off" meds were 5, 4 and 4. They all took carbidopa/L-dopa (137.5/850mg, $300 / 1200 \mathrm{mg}$, and $325 / 1300 \mathrm{mg}$ daily, respectively); 2 were on bromocriptine (\#1 - $11.25 \mathrm{mg}$, \#3 - $3.75 \mathrm{mg}$ daily); 1 was on selegiline (10 $\mathrm{mg} / \mathrm{d}) ; 2$ were on amantadine; and 1 was on ethopropazine.

Surgery was uneventful and post-operative $\mathrm{CT} / \mathrm{MR}$ scans revealed accurate placement of the grafts. There was no post-operative morbidity and all 3 patients were discharged 5-7 days post-op and maintained on their pre-op medication regimen.

Post-operative follow-up examinations were performed at 3 month intervals (\#1 - 3, 6, 9 mos.; \#2 - 3 and 6 mos.; \#3 - 3 mos.). Clinically 
all 3 patients developed a mild increase of dyskinesias starting around 2-3 mos. post-op without any change of medications (most notable in patients 2 and 3 ). The post-op UPDRS motor scores in the "off" state changed as follows: \#1 - 3 mo. increased 23\%, 6 mo. decreased 14\%, 9 mo. decreased 25\%; \#2 - 3 mo. decreased 25\%, 6 mo. decreased 58\%; \#3 3 mo. increased $10 \%$. Percent of waking day spent in the "off" condition changed as follows: \#1 - 3 mo. increased 50\%, 6 mo. decreased 0\%, 9 mo. decreased 50\%; \#2 - 3 mo. decreased $54 \%$ and 6 mo. decreased 54\%; \#3 - 3 mo. decreased $25 \%$. Timed motor performance tests at 6 mo. post-op in patients \#1 and 2 "off" meds revealed the following \% improvement: PRONSUP 18\% \#1, 44\% \#2; HAND-ARM 40\% \#1, $36 \%$ \#2; FINGER DEXT 15\% \#1, 23\% \#2; STAND-WALK-SIT 44\% \#1, 21\% \#2.

Movement time (MT-bradykinesia) studies "off" meds revealed $10 \%$ improvement at 9 mo. post-op for patient \#1 and 23\% improvement at 6 mo. post-op for patient \#2.

\section{SUMMARY}

1. Autologous intrastriatal adrenal medulla/ nerve cografting can be performed safely with careful patient selection and a strong medical/ surgical team approach.
2. Flank adrenalectomy and stereotaxic implantation significantly reduce post-operative morbidity and recovery time.

2. Early results are encouraging, but longer follow-up is needed. This procedure should still be considered investigational.

\section{REFERENCES}

1. Watts RL, Bakay RAE. Autologous adrenal medullato-caudate grafting for parkinsonism in humans and in nonhuman primates. Frontiers of Neuroendocrinology 1991; 12(No. 4): 357-378.

2. CAPIT Committee (Langston JW et al.) Core Assessment Program for Intracerebral Transplantations (CAPIT). Movement Disorders 1992; 7 (No. 1): 2-13.

3. Watts RL, Mandir AS, Ahn KJ, Juncos JL, Zakers GO, Freeman A. Electrophysiological analysis of early Parkinson's disease. Neurology 1991; 41 (Suppl 2): 4449. 

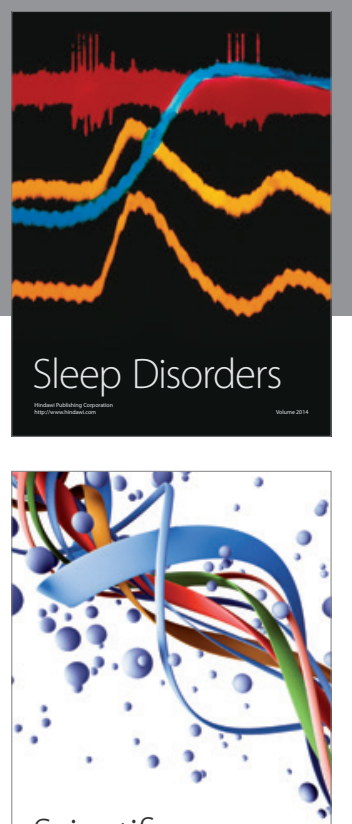

Scientifica
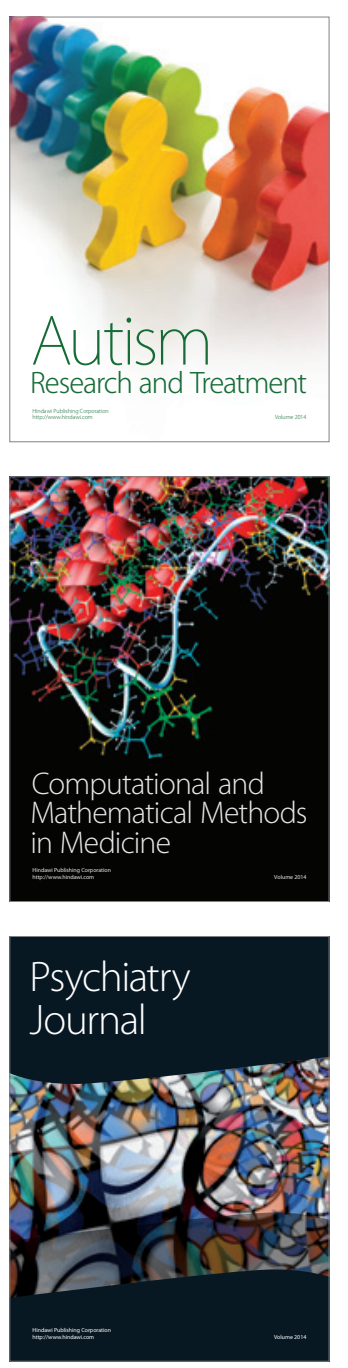
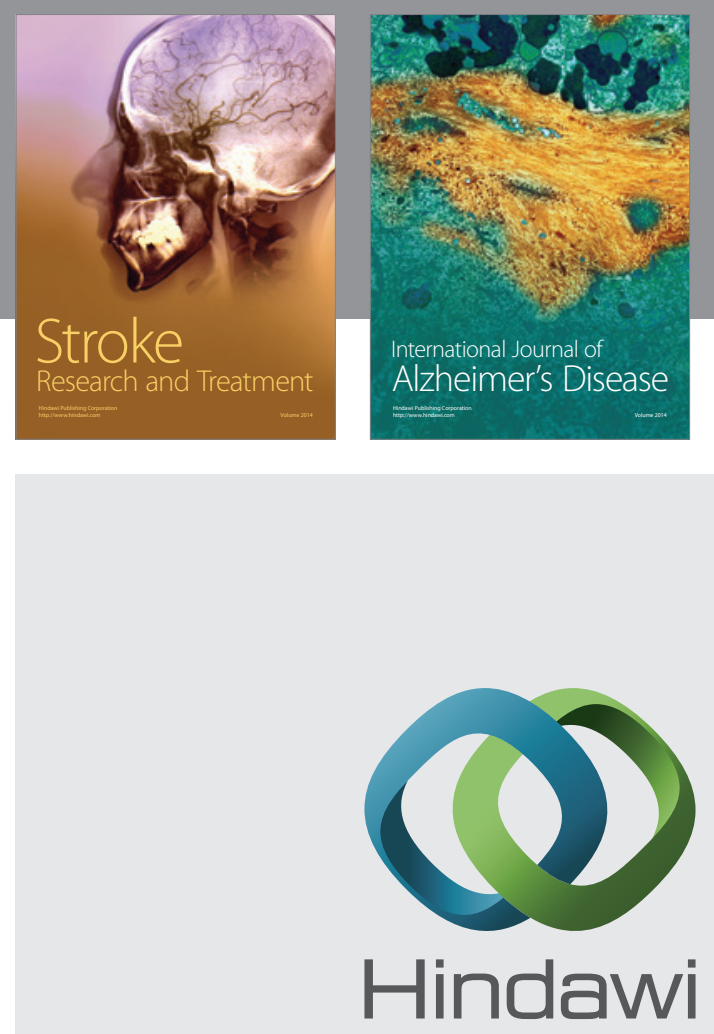

Submit your manuscripts at

http://www.hindawi.com
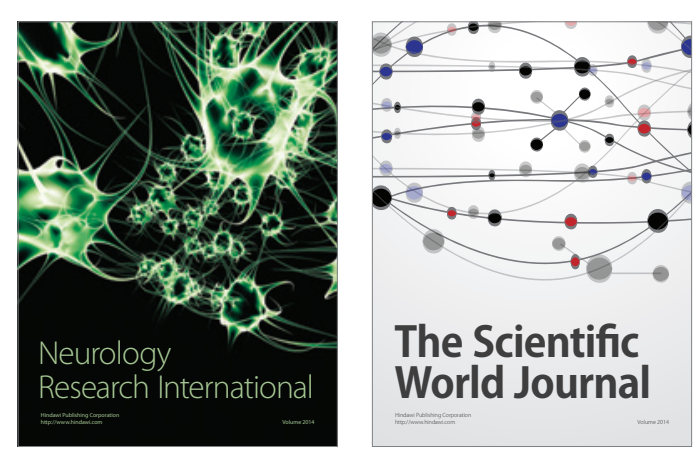

The Scientific World Journal

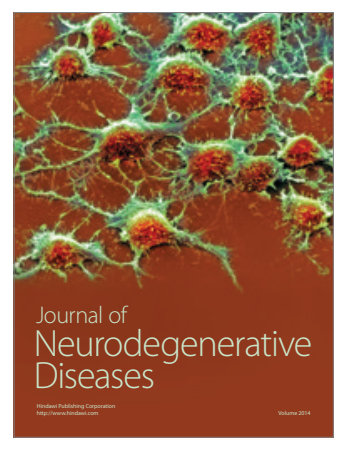

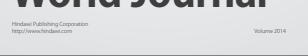

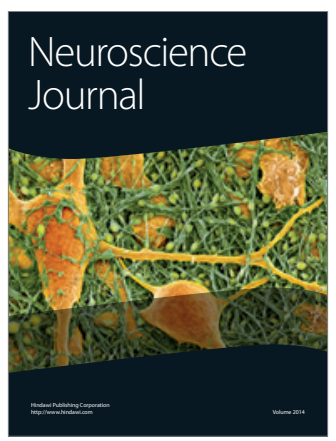

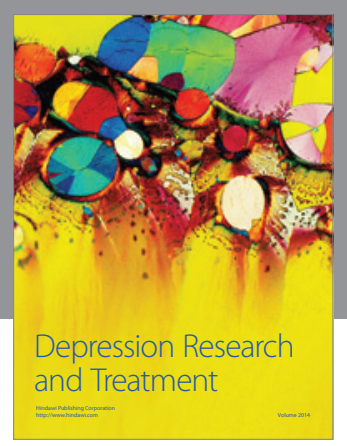
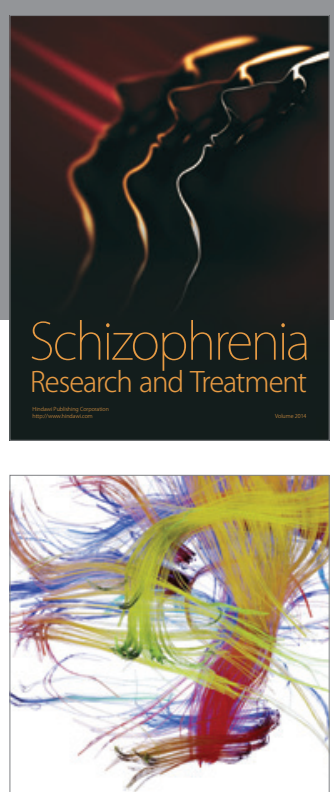

Brain Science

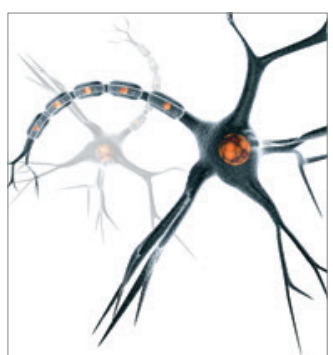

Neural Plasticity
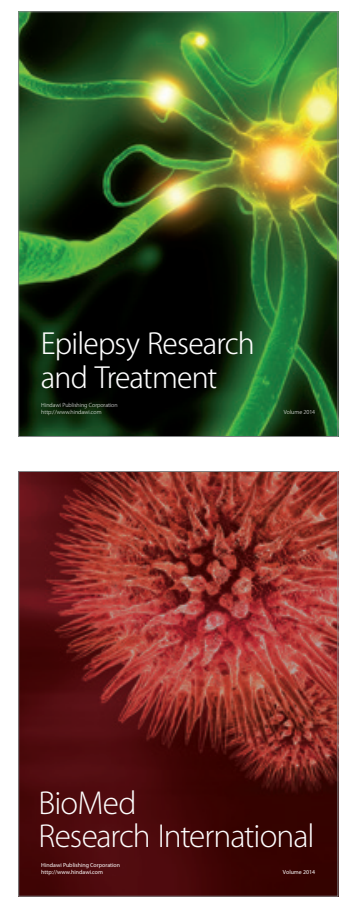

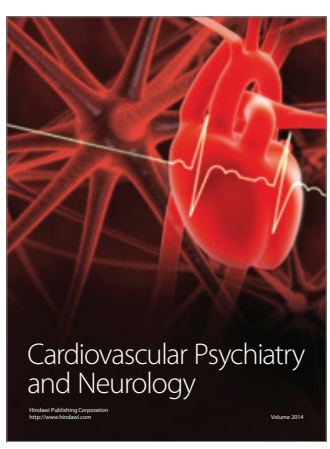

Parkinson's

Disease
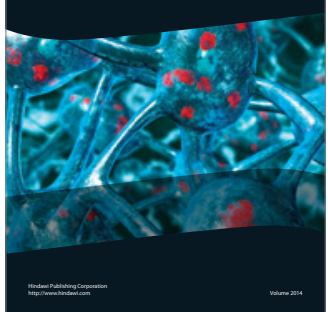